\title{
INFLUÊNCIA DO MOBILIÁRIO NAS ATIVIDADES LÚDICAS EM ESCOLARES COM PARALISIA CEREBRAL
}

\section{INFLUENCE OF THE FURNITURE IN THE PLAYFUL ACTIVITIES IN STUDENTS WITH CEREBRAL PALSY}

Oliveira FT, Braccialli LMP. Influência do Mobiliário nas Atividades Lúdicas em Escolares com Paralisia Cerebral. Rev Bras Crescimento Desenvolvimento Hum. 2008; 18(3): 308-320

\section{Resumo:}

Este trabalho analisou o desempenho de alunos com paralisia cerebral espástica durante a realização de atividades em dois tipos de assentos: lona e madeira. Participaram do estudo onze alunos com paralisia cerebral espástica, maiores de seis anos. Foi utilizado um protocolo de tarefas de membros superiores, adaptado para a realidade brasileira, para verificar se o assento utilizado na cadeira influenciava ou não o desempenho manual desses alunos. A coleta de dados realizou-se com os alunos sentados na cadeira com assento de lona, e na cadeira com o assento de madeira. A análise dos dados foi realizada por meio da pontuação das atividades realizadas nos dois assentos. Pôde-se concluir que todos os participantes apresentaram dificuldades na realização das atividades, devido às suas limitações motoras, visto que exigiam movimentos finos, força de preensão e coordenação, para sua realização efetiva. Foi possível observar, também, que a maioria dos participantes obteve maior pontuação no assento de lona. Além disso, pôde ser verificado que a distribuição topográfica da paralisia cerebral influenciou na realização das atividades.

Palavras-chave: Educação especial; paralisia cerebral; mobiliário; adaptação.

Trabalho baseado na dissertação: Estudo do mobiliário escolar durante o desempenho de atividades lúdicas por alunos com paralisia cerebral. Apoio financeiro: CAPES/ PROESP / SEESP / MEC / CNPq / Fundo de Pesquisa

* $\quad$ Fisioterapeuta. Mestre em Educação pela Unesp de Marília - SP. Programa de Pós-graduação em Educação pela Unesp de Marília - SP.

** Fisioterapeuta. Professora-doutora do Departamento de Educação Especial. Orientador do Programa de Pós-Graduação em Educação da Unesp de Marília - SP.

Correspondência para: Franciane Teixeira de Oliveira. R 15 de novembro, 159 - centro, CEP 17560-000, Vera Cruz - SP. Tel:(14) 34921010/(14)81495455. E-mail: fisiofran2003@yahoo.com.br 


\begin{abstract}
:
This work analyzed the performance of students with spastic cerebral palsy during the accomplishment of activities in two types of seats: canvas and wood. Eleven students with spastic cerebral palsy, bigger of six years had participated of the study. A protocol of tasks of superior members was used, adapted for the Brazilian reality, to verify if the seat used in the chair it influenced or not it manual performance of these students. The collection of data was become fullfilled with the students seated in the chair with canvas seat, and in the chair with the wooden seat. The analysis of the data was carried through by means of the punctuation of the activities carried through in the two seats. It could be concluded that all the participants had presented difficulties in the accomplishment of the activities, had to its motor limitations, since they demanded fine movements, force of grasp and coordination, for its accomplishment accomplishes. It was possible to observe, also, that the majority of the participants got greater punctuation in the canvas seat. Moreover, it could be verified that the topographical distribution of the cerebral paralysis influenced in the accomplishment of the activities.
\end{abstract}

Key words: Special education; cerebral palsy; furniture; adaptation.

\section{INTRODUÇÃO}

Vários autores afirmam que a paralisia cerebral pode ser definida como um grupo de desordens do desenvolvimento, do movimento e da postura, que causa limitações de atividades e dos movimentos voluntários; são atribuídas a distúrbios não progressivos que ocorreram no cérebro fetal ou infantil. As desordens motoras da paralisia cerebral freqüentemente estão acompanhadas por alterações sensoriais, na cognição, comunicação, percepção, comportamento e/ou crises convulsivas. ${ }^{1-5}$

Portanto, diante dos comprometimentos que as crianças com paralisia cerebral podem apresentar, tornam-se necessários ajustes na postura sentada. Uma postura sentada adequada melhora o desempenho de atividades funcionais de membros superiores ${ }^{6,7}$

Todavia, o sentar inadequado pode gerar encurtamentos e contraturas em flexão de musculatura posterior de membros superiores, luxação de quadril e deformidades posturais, o que costuma se agravar com o tempo e, com o crescimento do indivíduo. ${ }^{8}$
Portanto, a cadeira ideal para crianças com paralisia cerebral é aquela que proporciona maior estabilidade postural e, dessa maneira, maximiza o grau de independência funcional nos movimentos de braços e mãos. ${ }^{9}$ No entanto, o mobiliário ideal é aquele ajustado para cada indivíduo.

Em um trabalho, pesquisadores estudaram a atividade extensora muscular da musculatura posterior de membros inferiores de indivíduos com paralisia cerebral espástica, em diferentes posturas sentadas. Eles concluíram que a ação da musculatura extensora foi menor nos $0^{\circ}$ de inclinação do assento, com 90 graus de inclinação do encosto. ${ }^{10}$

Diferentemente, outros autores verificaram o efeito da inclinação do assento da cadeira na função de membros superiores de alunos com paralisia cerebral. Tais pesquisadores entraram em contradição, quando os autores do primeiro estudo observaram que não houve diferenças para a função das mãos, quando um indivíduo com paralisia cerebral se sentava com ângulo maior que $90^{\circ}$ de quadril. ${ }^{11,12}$

Outros autores verificaram, em seus estudos, que a inclinação do assento de 0 a 15 
graus anterior, melhorou o controle postural e, fez com que as mãos das crianças ficassem livres para realizar atividades. ${ }^{13-15}$

Certo estudo indicou que o uso da órtese abdutora e as inclinações do assento horizontal e inclinado anteriormente diminuem a atividade muscular dos membros inferiores e melhoram a função de membros superiores. ${ }^{16}$

Vários estudos mostraram efeitos positivos na função de membros superiores, em uma inclinação de neutra a leve $\left(10^{\circ}\right)$ da cadeira como um todo. O autor ainda constatou que, crianças com paralisia cerebral deveriam permanecer em cadeiras as quais oferecessem posição funcional: orientação no espaço de $0^{\circ}$ a $15^{\circ}$; utilização de faixas de quadril; apoio para pés; bandeja com recorte, assento inclinado anteriormente de 0 a $15^{\circ}$, para melhorar a função de membros superiores. ${ }^{17}$

Quanto aos acessórios e equipamentos utilizados na cadeira, foi observado que o sistema de assento que possuía suporte torácico e sacral se tornou efetivo na distribuição da massa corporal superior e melhorou significantemente a postura sentada de crianças com paralisia cerebral. ${ }^{18}$

Em relação ao tipo de assento, concluiuse que o assento em sela permitiu significante melhora no controle postural e, conseqüentemente, ajudou essas crianças a desenvolver e manter melhor controle postural e de movimentos de membros superiores. ${ }^{19}$

Em uma pesquisa foi verificado que o assento de espuma proporcionou uma superfície não escorregadia, em contraste com o assento de vinil. Os autores encontraram que as crianças avaliadas permaneceram cerca de $97 \%$ do tempo em alinhamento postural, na linha média no assento de espuma contornado. Em relação às atividades de membros superiores, os autores concluíram que as crianças ficavam mais independentes para brincar, no assento de espuma contornado. ${ }^{20}$
Com relação às diferentes posturas sentadas, estudos mostraram que a postura sentada com cifose, freqüentemente observada em crianças com paralisia cerebral, não é a causa de ajustes posturais anticonvencionais nessas crianças. Indicaram, ainda, que os ajustes posturais de crianças com paralisia cerebral espástica não dependem somente da postura sentada, mas também da severidade do quadro e de sua idade. ${ }^{21}$

Em relação à utilização de uma superfície de apoio para a realização de atividades, foi confirmado que a superfície de apoio influencia na extensão de membros superiores, o que resultou na melhora na realização da atividade. ${ }^{22}$

Um assento adequado contribui ativamente no desenvolvimento do controle postural da criança com paralisia cerebral. Isso deveria permitir às crianças o dinamismo e um alto grau da habilidade do sentar. O assento é parte integrante no auxílio do controle postural sentado de uma criança com paralisia cerebral. ${ }^{23}$

A alteração da estrutura do assento é um fator muito importante na indicação de superfícies de suporte. O assento inflexível é incapaz de mudar sua forma, por isso a pressão externa pode comprimir e deformar tecidos e vasos, e provocar uma necrose. Por sua vez, o assento flexível pode causar instabilidade postural e inabilidade do uso de membros superiores. ${ }^{24}$

Portanto, diante dessa situação, surgiu o problema: o assento da cadeira realmente influencia o desempenho de membros superiores, em atividades escolares de alunos com paralisia cerebral?

Por conseguinte, o objetivo deste trabalho foi analisar o desempenho de alunos com paralisia cerebral espástica, durante a realização de atividades, em dois tipos de assentos: de lona e de madeira. 


\section{MÉTODO}

O trabalho foi encaminhado ao Comitê de Ética em Pesquisa da Faculdade de Filosofia e Ciências, Unesp - Marília, e aprovado sob o parecer $n^{\circ}$. 2692/2006. Os responsáveis pelos participantes assinaram o Termo de Consentimento Livre e Esclarecido para a participação voluntária na pesquisa.

A pesquisa foi realizada no Laboratório de Análise de Movimento do Centro de Estudos da Educação e da Saúde (CEES), unidade auxiliar da Faculdade de Filosofia e Ciências, Unesp - Marília, no estado de São Paulo - Brasil. O tempo de avaliação de cada participante foi cerca de 10 minutos em cada assento.

Foram adotados como critérios de inclusão de participantes: ter idade acima de seis anos; ter boa compreensão, para entender as atividades propostas; ter bom controle de tronco; ter habilidades motoras em pelo menos um dos membros superiores, necessárias para a execução das atividades; e não ter déficits de acuidade visual acentuados. Os critérios de exclusão foram: ter diagnóstico de baixa visão ou cegueira, e possuir deformidades nos dois membros superiores.
Todas as informações sobre as características dos participantes foram obtidas nos prontuários existentes no Centro de Estudos da Educação e da Saúde (CEES) e em outras instituições nas quais os participantes eram atendidos.

Foram participantes 11 alunos com paralisia cerebral espástica, de ambos os sexos, com idades entre 7 e 28 anos (Quadro 1).

Para a coleta de dados, primeiramente houve a seleção dos participantes e foi realizada uma avaliação antropométrica.

Em seguida, foi construída uma cadeira de madeira com assento de lona, com regulagem de altura para o apoio de pés e abdutor, e de profundidade, para o assento.

Em relação ao assento, a cadeira foi construída com um assento fixo de lona, semelhante ao de cadeiras de rodas disponíveis no mercado. Foi confeccionado, também, um assento de madeira removível que podia ser encaixado em cima do assento de lona, conforme a situação experimental de coleta de dados (Figura 1).

Como instrumentos para coleta de dados foram utilizadas as Tarefas de Controle Motor de Membros Superiores ${ }^{25}$, adaptadas à realidade brasileira. Os autores das tare-

Quadro 1: Caracterização dos participantes do estudo

\begin{tabular}{lllllll}
\hline Participantes & Idade & Gênero & Cor & $\begin{array}{l}\text { Distribuição topográfica da } \\
\text { paralisia cerebral }\end{array}$ & $\begin{array}{l}\text { Data da } \\
\text { avaliação }\end{array}$ & $\begin{array}{l}\text { Membro } \\
\text { superior } \\
\text { que usou }\end{array}$ \\
\hline P1 & 12 anos & Feminino & Branca & Diparesia espástica & $12 / 12 / 2006$ & $\begin{array}{l}\text { Direito } \\
\text { P2 }\end{array}$ \\
& 20 anos & Masculino & Branca & Diparesia espástica & $12 / 12 / 2006$ & Direito \\
P3 & 28 anos & Masculino & Branca & Quadriparesia espástica & $13 / 12 / 2006$ & Esquerdo \\
P4 & 10 anos & Feminino & Branca & Hemiparesia espástica á direita & $13 / 12 / 12006$ & Esquerdo \\
P5 & 7 anos & Feminino & Branca & Diparesia espástica & $13 / 12 / 1006$ & Direito \\
P6 & 24 anos & Masculino & Branca & Quadriparesia espástica & $14 / 12 / 2006$ & Direito \\
P7 & 15 anos & Masculino & Negro & Diparesia espástica & $14 / 12 / 2006$ & Esquerdo \\
P8 & 22 anos & Feminino & Branca & Diparesia espástica & $14 / 12 / 2006$ & Esquerdo \\
P9 & 14 anos & Masculino & Branca & Hemiparesia espástica á esquerda & $15 / 12 / 2006$ & Direito \\
P10 & 9 anos & Masculino & Branca & Hemiparesia espástica á direita & $15 / 12 / 1006$ & Esquerdo \\
P11 & 21 anos & Masculino & Branca & Diparesia espástica & $15 / 12 / 2006$ & Direito \\
\hline
\end{tabular}


Figura 1: Cadeira com assento fixo de lona, assento removível de madeira, com regulagem de apoio de pés, abdutor de quadril e profundidade do assento

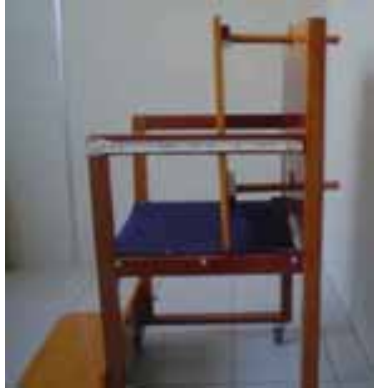

fas foram contatados via e-mail e autorizaram o uso e a adaptação do protocolo (Quadro 2).

A pontuação das atividades foi atribuída de acordo com cada atividade: as atividades de dar toques com os dedos linear e em curva foram pontuadas pelo número de gols que o participante conseguiu realizar em um tempo de 10 segundos; as atividades de pegar

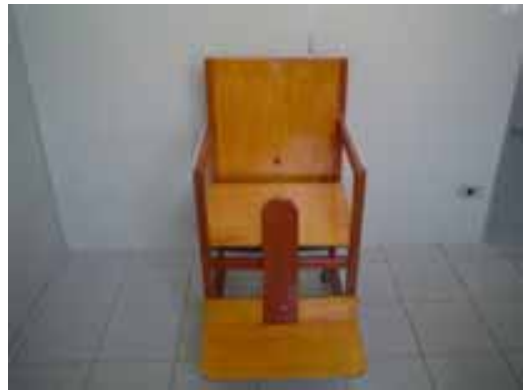

bolinhas de gude e do prendedor de roupa, pelo tempo total que o participante demorou para completar a atividade; a atividade de pressionar com o polegar, pelo número de vezes que o participante acendeu a luz, por meio do acionador, com o polegar, enquanto a atividade do traçado com o lápis foi pontuada pelo tempo total que o participante demorou para completar a atividade.

\section{Quadro 2: Adaptação das tarefas de controle motor de membros superiores}

\begin{tabular}{|c|c|c|}
\hline Atividade & Descrição & $\begin{array}{c}\text { Mensuração } \\
\text { da performance }\end{array}$ \\
\hline 1 - Dar toques com os dedos linear & $\begin{array}{l}\text { Deslocar uma bolinha } \\
\text { de futebol de botão por } \\
\text { meio de toques com os } \\
\text { dedos alternados até } \\
\text { atingir o gol, no alcan- } \\
\text { ce máximo da mesa, na } \\
\text { frente do participante. }\end{array}$ & $\begin{array}{l}\text { Número de gols } \\
\text { em } 10 \text { segundos }\end{array}$ \\
\hline 1 - Dar toques com dedo em curva & $\begin{array}{l}\text { Deslocar uma bolinha } \\
\text { de futebol de botão por } \\
\text { meio de toques com os } \\
\text { dedos alternados, com } \\
\text { dois obstáculos coloca- } \\
\text { dos entre a criança e o } \\
\text { gol, fazendo com que a } \\
\text { mão do participante se } \\
\text { mova em um trilha com } \\
\text { curvas. }\end{array}$ & $\begin{array}{l}\text { Número de gols } \\
\text { em } 10 \text { segundos }\end{array}$ \\
\hline
\end{tabular}




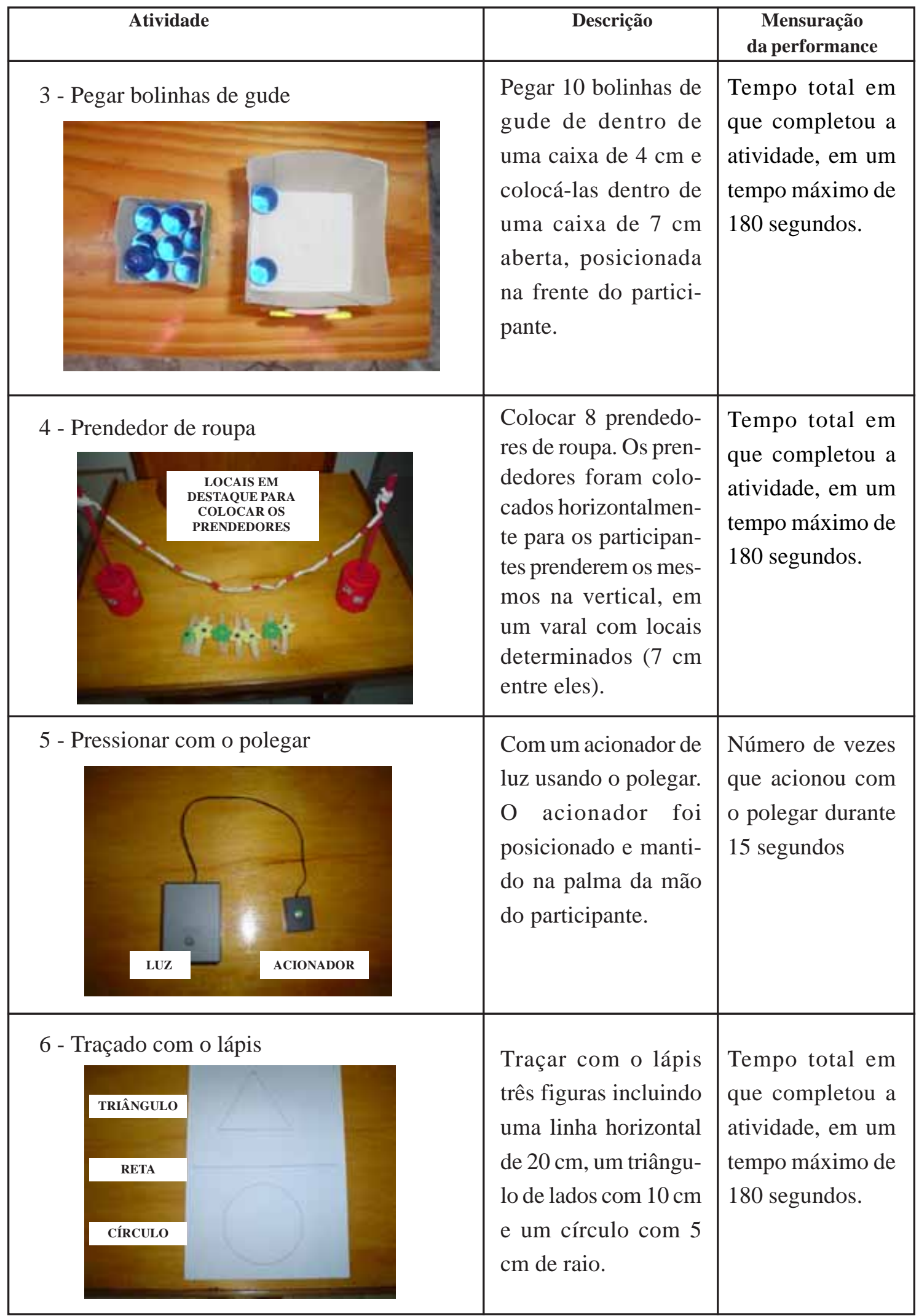


A coleta de dados constou de duas situações experimentais: 1) Assento rígido de madeira, e 2) assento flexível de lona.

Nas duas situações o participante era posicionado na cadeira, e esperava-se cerca de três minutos para que adequasse sua postura. ${ }^{26,27}$ Em seguida, a pesquisadora instruía o participante em relação às atividades. O participante iniciava cada atividade ao sinal da pesquisadora.

O estudo foi randômico, por sorteio, feito antes da coleta de dados, sendo os dados anotados na ficha do participante, para que fosse diminuída a porcentagem de erros e para que os possíveis erros que pudessem ocorrer não se devessem à aprendizagem da atividade em determinado tipo de assento.

A pontuação das atividades era anotada pela pesquisadora e por um auxiliar de coleta de dados, na ficha de cada participante.

O desempenho dos alunos foi analisado por meio da pontuação das atividades realizadas pelos participantes. Todas as atividades foram pontuadas, tendo sido examinado se o tipo de assento utilizado influenciou no desempenho das atividades.

Para a análise dos dados, fez-se uma mensuração da pontuação em cada atividade.
Cada atividade foi pontuada de acordo com a complexidade de sua realização.

A soma das seis atividades totalizaria no máximo 100 pontos. As mensurações foram efetuadas para os dois assentos, em todas as atividades.

\section{RESULTADOS E DISCUSSÃO}

Sete participantes realizaram as atividades, primeiramente no assento de madeira: P2, P3, P6, P7, P8, P9 e P11. Quatro participantes começaram no assento de lona: P1, P4, P5 e $\mathrm{P} 10$. A seqüência dos assentos foi disposta aleatoriamente.

Foi feita a soma da pontuação dos participantes nas seis atividades, nos assentos de lona e madeira, para comparar em qual assento os alunos obtiveram melhor desempenho (Tabela 1).

De acordo com a Tabela 1, pode-se observar que os participantes P1, P4 e P10 tiveram menor pontuação, no assento de lona. Portanto, seu melhor desempenho foi no assento de madeira.

Os participantes P2, P3, P5, P6, P7, P9 e P11 tiveram maior pontuação, no assento de

Tabela 1: Pontuação total das seis atividades dos participantes nos assentos de lona e madeira

\begin{tabular}{ccc}
\hline Participantes & Pontos na lona & Pontos na madeira \\
\hline P1 & 97,5 & 100 \\
P2 & 80 & 77,5 \\
P3 & 22,5 & 20 \\
P4 & 35 & 47,5 \\
P5 & 67,5 & 65 \\
P6 & 87,5 & 85 \\
P7 & 20 & 12,5 \\
P8 & 62,5 & 62,5 \\
P9 & 75 & 72,5 \\
P10 & 87,5 & 92,5 \\
P11 & 52,5 & 50 \\
\hline
\end{tabular}


lona, no qual mostraram melhor desempenho. Somente o participante P8 obteve igual pontuação em ambos os assentos.

Esse fato contradiz os relatos encontrados na literatura, que dão conta de que, em assentos flexíveis, o tronco de indivíduos com prejuízos motores tende a ficar instável, o que prejudica funções como as de membros superiores. $^{28,29}$

Porém, a ordem dos assentos parece ter interferido na pontuação total dos participantes. O que pôde ser observado foi que P2, P3, P6, P7, P9 e P11, os quais começaram as atividades no assento de madeira, tiveram maior pontuação no assento de lona. E os participantes P1, P4 e P10, que começaram as atividades no assento de lona, obtiveram maior pontuação no assento de madeira, com exceção do P8 que pontuou igualmente nos dois assentos e P5, que começou as atividades no assento de lona e pontuou melhor neste mesmo assento.

Tal situação também se mostrou contraditória, pois, foi realizado um sorteio de escolha dos assentos para que não houvesse erros, em relação à aprendizagem das atividades com a ordem dos assentos. Além disso, os alunos foram orientados quanto à realização das atividades antes da coleta de dados e estavam familiarizados com elas. Ainda, a aprendizagem, de um modo imediato, de alunos com paralisia cerebral pode estar dificultada pelos seus déficits motores.

Verificou-se, ainda, nos resultados, que a maioria dos participantes teve dificuldades na realização das atividades propostas, como foi mostrado na Tabela 1. As principais limitações nos indivíduos com paralisia cerebral, as quais podem acarretar problemas durante a execução de atividades manuais com os membros superiores, estão relacionadas ao alcance, preensão e manipulação. Tais limitações podem ser decorrentes de comprometimentos motores, sensoriais e perceptuais. Entre os comprometimentos neuromusculares, podem ser mencionados a fraqueza, a espasticidade, as sinergias anormais, o desequilíbrio muscular e a incoordenação. ${ }^{30}$

As dificuldades encontradas na realização de atividades pelas crianças com paralisia cerebral também podem ter origem cognitiva. A paralisia cerebral pode vir acompanhada de déficits de atenção e aprendizagem.

Os participantes P4 e P10, que eram hemiparéticos, obtiveram uma pontuação ainda menor que o participante $\mathrm{P} 1$, que era diplégico.

Em uma pesquisa, 30\% das crianças hemiplégicas tinham limitações no membro superior não afetado, enquanto $70 \%$ dos diplégicos exibiam déficits nos dois membros superiores. ${ }^{29}$ Outro estudo comparou crianças hemiplégicas e diplégicas, e concluiu que há acentuadas diferenças entre elas, em vários aspectos motores, na realização e participação de atividades e na qualidade de vida dessas crianças. ${ }^{31}$

Por outro lado, P6 também tinha paralisia cerebral do tipo quadriparesia espástica. No entanto, ele obteve pontuação alta. Tal fato é contraditório com respeito à literatura encontrada, visto que os trabalhos indicam que indivíduos com quadros mais graves apresentam desempenho pior de membros superiores. Porém, ele pode ter-se diferenciado dos outros participantes, pelo seu grau de espasticidade, o que não foi medido neste estudo. A diferença pode ainda ser explicada pela diferença nas limitações cognitivas que existem entre os indivíduos com paralisia cerebral.

Resultados e discussão do desempenho dos participantes em cada atividade nos assentos de lona e de madeira

Os participantes P3, P4, P6, P9 e P11 tiveram maior pontuação no assento de lona, na atividade de dar toques com os dedos linear. Os participantes P3, P6, P9 e P11 começaram as atividades no assento de madeira e P4, no assento de lona.

Nessa atividade, os participantes P2 e P10 obtiveram maior pontuação no assento de 
madeira. O P2 começou as atividades no assento de madeira e P10, no de lona. Os participantes $\mathrm{P} 1, \mathrm{P} 5, \mathrm{P} 7, \mathrm{P} 8$ pontuaram de modo igual, nos dois tipos de assentos.

A pontuação na atividade de dar toques com os dedos em curva foi semelhante para todos os participantes, nos dois tipos de assentos.

Os participantes P1, P5 e P10 pontuaram melhor no assento de madeira. Esses participantes começaram as atividades no assento de lona. Os outros participantes obtiveram a mesma pontuação, nos dois assentos. Nenhum participante pontuou zero nessa atividade.

Os participantes que tiveram menor pontuação, tanto no assento de lona como no de madeira, foram P3, P4, P7 e P11. As maiores pontuações foram de P1, P2 e P10.

As atividades de dar toques com os dedos linear e em curva exigiam planejamento e coordenação motora fina, para sua realização. Apesar de alguns participantes realizarem a pontuação máxima, nessas duas atividades, a maioria deles encontrou dificuldades. De sorte, a corroborar tais achados, certo estudo indicou que durante a realização de atividades que exigem manipulação motora fina, as crianças com paralisia cerebral geralmente empregam vários dedos e realizam movimentos lentos e desajeitados. ${ }^{33}$ Já outro estudo enfatizou que crianças com paralisia cerebral possuem menor habilidade de aprender movimentos novos e têm diminuição da velocidade dos movimentos. $^{34}$

Além disso, essas crianças podem apresentar déficits de planejamento, de controle e de cognição, o que prejudica a realização adequada das atividades. Estratégias cognitivas são baseadas em experiências passadas, que nem sempre são oferecidas às crianças com paralisia cerebral, devido às suas limitações motoras. ${ }^{35}$ Porém, quando o indivíduo é submetido a experiências anteriores, ele pode se utilizar de estratégias adaptativas, para a realização da atividade. ${ }^{36}$
Assim, os dados do estudo realizado condizem com a literatura, uma vez que os participantes que tiveram menor desempenho, nessas atividades, tinham um maior comprometimento motor. Em relação ao déficit cognitivo, sabe-se que ele influencia na realização de atividades, todavia, isso não pôde ser comprovado neste trabalho, visto que não foram feitos testes para avaliação do aspecto cognitivo dos participantes.

$\mathrm{Na}$ atividade de pegar bolinhas de gude, nenhum participante obteve melhor desempenho no assento de lona.

Os participantes $\mathrm{P} 4$ e P5 obtiveram melhor desempenho no assento de madeira. Esses participantes realizaram as atividades primeiramente no assento de lona. Os outros participantes obtiveram a mesma pontuação, nos dois assentos. O participante P7 não pontuou nos dois tipos de assentos.

Os participantes P1, P2, P6, P8, P9 e P10 obtiveram pontuação máxima nessa atividade, nos dois assentos utilizados. Os participantes P1, P2 e P8 apresentavam diparesia espástica, P6 apresentava quadriparesia espástica, e P9 e P10 apresentavam hemiparesia.

$\mathrm{Na}$ atividade de pegar bolinhas de gude, a preensão de precisão era necessária para a sua realização.

A preensão é uma habilidade motora delicada, caracterizada pelo emprego das mãos e dos dedos, na aproximação preensora dos objetos e nos atos de pegá-los e manipulá-los.

A maioria dos participantes deste estudo apresentou dificuldades na realização da atividade de pegar bolinhas de gude, o que condiz com a literatura, a qual indica que indivíduos com paralisia cerebral possuem dificuldades na realização da preensão precisa. ${ }^{37}$ Crianças com paralisia cerebral, principalmente com hemiplegia, apresentam disfunção na preensão, como conseqüência de lesões no córtex sensório-motor e no tracto corticoespinhal. ${ }^{33}$ 
No entanto, o fato dos participantes com diferentes tipos de paralisia cerebral obterem o mesmo desempenho pode depender do grau de espasticidade e de habilidade de cada um. E, ainda, sabe-se que um mesmo quadro de paralisia cerebral pode apresentar várias diferenças. ${ }^{38}$

O participante $\mathrm{P} 5$ obteve uma maior pontuação no assento de lona, na atividade do prendedor de roupa. Por sua vez, o participante P10 teve uma maior pontuação no assento de madeira, nessa mesma atividade. Os dois participantes começaram as atividades no assento de lona.

Os participantes P3, P4 e P7 não pontuaram nos dois tipos de assentos.

Os participantes P1 e P2 tiveram pontuação máxima nos dois assentos nessa atividade. Esses participantes apresentavam diparesia espástica.

Na atividade do prendedor de roupa, os participantes necessitavam realizar uma preensão digital. Existem três tipos de preensões: 1) preensões digitais, nas quais se emprega apenas os dedos para a preensão; 2) preensões palmares, que envolvem a participação dos dedos e da palma da mão; 3) preensões centradas, que possibilitam uma simetria do eixo longitudinal da mão. ${ }^{39}$

Além disso, eles necessitavam ter força nas pontas dos dedos para apertar o prendedor. Como já se mencionou, anteriormente, a preensão é um movimento de difícil realização para indivíduos com paralisia cerebral, visto que apresentam dificuldades na quantidade de força que devem aplicar, para pegar ou apertar um objeto. ${ }^{40}$

Os participantes P2, P5 e P10 tiveram um melhor desempenho no assento de lona, na atividade de pressionar com o polegar. O participante P2 começou a realizar as atividades no assento de madeira, enquanto P5 e P10 começaram no assento de lona. Os demais realizaram a atividade sem diferenças de pontuação, nos dois tipos de assentos. Os participantes P3, P4 e P7 pontuaram zero, em ambos os ti- pos de assentos. Os participantes P1, P6 e P9 obtiveram a nota máxima, nos dois tipos.

A atividade de pressionar com o polegar exigia um movimento de oponência do polegar. Sabe-se que há limitações na dissociação de dedos, em crianças com paralisia cerebral, assim como a lentidão, fraqueza e movimentos incoordenados de membros superiores. ${ }^{41}$

As principais musculaturas que sofrem com a espasticidade em membros superiores são flexores de ombro, extensores de cotovelo, extensores de punho e de dedos. ${ }^{1,42}$

Portanto, o punho desses indivíduos tende a ficar flexionado, o que impede a extensão e dissociação dos dedos, para realização da oponência do polegar, o que pode justificar os resultados deste estudo.

Os participantes $\mathrm{P} 4$ e $\mathrm{P} 5$ tiveram maior pontuação, no assento de madeira, na atividade do traçado com o lápis. Esses participantes começaram as atividades no assento de lona. O participante $\mathrm{P} 7$ teve melhor desempenho no assento de lona e realizou as atividades primeiramente no assento de madeira. Os outros participantes pontuaram de modo igual, nos dois tipos de assentos.

Na atividade do traçado com o lápis, nenhum participante obteve zero na pontuação; ressalte-se que, os participantes P1, P6 e P10 tiveram a pontuação máxima (30 pontos), em ambos os tipos de assento.

O participante que menor pontuou, nessa atividade, foi o P3, que tinha paralisia cerebral do tipo quadriparesia espástica.

Para fazer a atividade do traçado com o lápis, era necessária coordenação e força de preensão digital. Esses movimentos são realizados com dificuldades por indivíduos com paralisia cerebral, devido às limitações motoras, sensoriais, perceptuais e de planejamento que costumam apresentar. Além disso, indivíduos com paralisia cerebral realizam esses movimentos mais lentos, com maior amplitude e com menor acurácia. $^{44}$ 
Os participantes apresentaram dificuldades na realização das atividades, devido às suas limitações motoras. As duas atividades em que os participantes tiveram maiores dificuldades foram: do prendedor de roupa e do traçado com o lápis, visto que estas exigiram movimentos finos, força de preensão e coordenação, para sua realização efetiva.

Foi possível observar, também, que a maioria dos participantes obteve maior pontuação no assento de lona, fato contraditório

\section{REFERÊNCIAS}

1. Souza, AMC, Ferrareto, I. Paralisia cerebral: aspectos práticos. São Paulo: Memnon; 1998.

2. Bax, M. Aspectos clínicos da paralisia cerebral. In: Finnie, NR, editor. O manuseio em casa da criança com paralisia cerebral. 1er ed. São Paulo: Manole; 2000. p.8-18.

3. Lebiedowska, MK, Gaebler-Spira, D, Burns, RS, Fisk, JR. Biomechanics characteristics of patients with spastic and dystonic hypertonia in cerebral palsy. Arch Phys Med Rehabil. 2004;85: 875-880.

4. Gauzzi, LDV, Fonseca, LF. Classificação da Paralisia Cerebral. In: Lima, CLA, Fonseca, LF. editores. Paralisia Cerebral: neurologia, ortopedia e reabilitação. Rio de Janeiro:Guanabara Koogan;2004.p.37-44.

5. Rosenbaum, P, Dan, B, Leviton, A, Paneth, N, Jacobsson, B, Goldstein, M. et al. Definition and Classification of Cerebral Palsy. 2005. [acesso em 11 jun 2007]. Disponível em: http://www.castangfoundation.net/docs/ washington_FINALManuscript.doc

6. Braccialli, LMP, Baraúna, MA. Contribuição da fisioterapia no sentar da criança com paralisia cerebral: um com relação à literatura, uma vez que é encontrado que, quanto mais instável a superfície de assento, pior a função de membros superiores. No entanto, a pontuação total das atividades parece ter sido influenciada pela ordem dos assentos, o que não aconteceu quando se observou a pontuação individual por atividade.

Além disso, pode ser verificado que a distribuição topográfica da paralisia cerebral influenciou na realização das atividades.

estudo de caso. Temas sobre

Desenvolvimento.2002;11(62):56-58.

7. Bain, DS, Ferguson-Pell, M. Remote monitoring of sitting behavior of people with spinal cord injury. J Rehabil Research Develop.2002;39(4):513-520.

8. Braccialli, LMP. Influência da utilização do mobiliário adaptado na postura sentada de indivíduos com paralisia cerebral espástica [tese]. Campinas:Faculdade de Educação Física da Universidade Estadual de Campinas; 2000.

9. Myhr, U, Wendt, LV. Improvement of functional sitting position for children with cerebral palsy. Develop Med \& Child Neurol.1991;33:246-256.

10. Nwaobi, OM, Brubaker, CE, Cusick, B, Sussman, M. Electromyographic investigation of extensor activity in cerebral-palsied children in different seating positions. Develop Med \& Child Neurology.1983;25:175-183.

11. Seeger, BR, Caudrey, DJ, O’mara, NA. Hand function in cerebral palsy: the effect of hip-flexion angle. Develop Med \& Child Neurology. 1984;26:601-606.

12 Nwaobi, OM. Seating orientations and upper function in children with cerebral palsy. Physical

Therapy.1987;67(8):1209-1212.

13. Myhr, U, Wendt, LV. Improvement of functional sitting position for children 
with cerebral palsy. DevelopMed \& Child Neurology.1991;33: 246-256.

14. Sochaniwskyj, A, Koheil, R, Bablich, K, Milner, M, Lotto, W. Dynamic monitoring of sitting posture for children with spastic cerebral palsy. Clin Biomechan.1991;6(3):161-167.

15. Reid, DT, Sochaniwskyj, A, Milner, M. Instrumentation and a protocol for quantification of upper-limb movement of children with and without cerebral palsy in two sitting positions. J Neurol Rehabil.1992;6: 25-34.

16. Myhr, U, Wendt, LV. Influence of different sitting positions and abduction orthoses on leg muscle activity in children with cerebral palsy. Develop Med \& Child Neurology.1993; 35(10): 870-880.

17. Stavness, C. The effect of positioning for children with cerebral palsy on upperextremity function: a review of evidence. Physic Occup Therapy in Pediatrics.2006; 26(3):39-53.

18. Dupuis, CC, Hoshizaki, TB, Gledhill, R, Batisita, WC. Uma comparação biomecânica de dois sistemas de assento para crianças portadoras de paralisia cerebral moderada. Revista Brasileira de Ciência e Movimento.1991; 5: 22-30.

19. Reid, DT. The effects of the saddle seat on seated postural control and upperextremity movement in children with cerebral palsy. Develop Med \& Child Neurology.1996;38:805-815.

20. Washington, K, Deitz, JC, White, OR, Schwartz, IS. The effects of a contoured foam seat on postural alignment and upper-extremity function in infants with neuromotor impairments. Physical Therapy. 2002; 82(11):1064-1076.

21. Brogren, E, Forssberg, H, HaddersAlgra, M. Influence of two different sitting positions on postural adjustments in children with spastic diplegia.
Developmental Medicine \& Child Neurolog. 2001; 43:534-546.

22. Utley, A. The influence of perturbing the working surface during reaching and grasping in children with hemiplegic cerebral palsy. Disabil Rehabil. 2007; 29(1):11-79.

23. Green, EM, Nelham, RL. Development of sitting ability, assessment of children with a motor handicap and prescription of appropriate seating systems.

Prosthetics and Orthotics Internat. 1991; 15:203-216.

24. Kochhann, ARS, Canali, N, Serafim, MAP. Comparação de picos de pressão em assento flexível em portadores de lesão medular e indivíduos normais: uma avaliação por interface de pressão. Acta Fisiátrica. 2004; 11(3): 95-100.

25. Mcclenaghan, BA, Thombs, L, Milner, M. Effects of seat-surface inclination on postural stability and function of the upper extremities of children with cerebral palsy. Develop Med \& Child Neurology. 1992; 34: 40-48.

26. Aissaoui, R, Coucher, C, Bourbonnais, D, Lacoste, M, Dansereau, J. Effect of seat cushion on dynamic stability in sitting during a reaching task in wheelchair users with paraplegia. Archives of Physical Medicine and Rehabilitation.2001 Feb; 82:274-281.

27. Myhr, U, Wendt, LV, Norrlin, S, Radell, U. Five-year follow-up of function sitting position in children with cerebral palsy. Develop Med \& Child Neurology. 1995; 37: 587-596.

28. Lanzetta, D, Cattaneo, D, Pellegatta, D, Cardini, R. Trunk control in unstable sitting posture during functional activities in healthy subjects and patients with multiple sclerosis. Archives of Physical Medicine and Rehabilitation. 2004 Feb; 85: 279-283. 
29. Lacoste, M, Therrien, M, Côté, JN, Shrier, I, Labelle, H, Prince, F.

Assessment of seated postural control in children: comparison of a force platform versus a pressure mapping system. Arch Physic Med and Rehabil. 2006 Dec; 87: 1623-1629.

30. Finnie, NR. O manuseio em casa da criança com paralisia cerebral. São Paulo: Manole, 2000.

31. Damiano, D, Abel, M, Romness, M, Tylkowsky, C, Gorton, G, Bagley, A. et al. Comparing functional profiles of children with hemiplegic and diplegic cerebral palsy in GMFCS levels I and II: are separate needed? Develop Med \& Child Neurology. 2006; 48: 497-803.

33. Harbourne, RT. Accuracy of movement speed and error detection skills in adolescents with cerebral palsy. Perceptual and Motor Skills. 2001; 93(2): 419-431.

34. Thorpe, DE, Valvano, J. The effects of knowledge of performance and cognitive strategies on motor skill learning in children with cerebral palsy. Pediatric Physic Therapy. 2002; 14(1): 2-15.

35. Steenbergen, B, Utley, A. Cerebral palsy: recent insights into movement deviations. Motor Control. 2005; 9: 353-356.

36. Brown, JK, Rensburg, VE, Walsh, G, Lakie, M, Wright, GW. A neurological study of hand function of hemiplegic children. Develop Med \& Child Neurology. 1987; 29: 287-304.
37. Gordon, AM, Duff, SV. Relation between clinical measures and fine manipulative control in children with hemiplegic cerebral palsy. Develop Med \& Child Neurology. 1999; 41: 856-891.

38. Palisano, RJ, Cameron, D, Rosenbaum, PL, Walter, SD, Russel, D. Stability of the Gross Motor Function Classification System. Develop Med \& Child Neurolog.2006; 48: 424-428.

39. Kapandji, AI. Fisiologia articular: esquemas comentados de mecânica humana. Rio de Janeiro:Guanabara Koogan, 2000.

40. Gordon, AM, Charles, J, Duff, SV. Fingertip forces during object manipulation in children with hemiplegic cerebral palsy. II: bilateral coordination. Develop Med \& Child Neurology. 1999; 41: 176-185.

41. Eliasson, AC, Forssberg, H, Hung, YC, Gordon, AM. Development of hand function and precision grip control in individuals with cerebral palsy: a 13year follow-up study. Pediatrics. 2006 October; 118(4): e1226-e1236.

42. Potén, E, Fridén, J, Thornell, L-E, Lieber, RL. Spastic wrist flexors are more severely affected than wrist extensors in children with cerebral palsy. Develop Medic \& Child Neurology. 2005; 47: 384-389.

43. Volman, MJM. Spatial coupling in children with hemiplegic cerebral palsy during bimanual circle and line drawing. Motor Control. 2005; 9: 395-416.

Recebido em: 30/03/2008 Modificado em: 19/06/2008 Aceito em: 30/11/2008 